\title{
RNAi-mediated downregulation of DNA binding protein A inhibits tumorigenesis in colorectal cancer
}

\author{
RUI-TING LIU ${ }^{1,2}$, GUO-RONG WANG ${ }^{2}$, CHANG LIU $^{1}$, JIAN QIU ${ }^{2}$, LI-KUN YAN $^{2}$, \\ XIAO-JUN LI ${ }^{2}$ and XIAO-QIANG WANG ${ }^{2}$ \\ ${ }^{1}$ Department of Hepatobiliary Surgery, The First Affiliated Hospital, College of Medicine, Xi'an Jiaotong University, \\ Xi'an, Shaanxi 710061; ${ }^{2}$ Department of General Surgery, The Third Affiliated Hospital, College of Medicine, \\ Xi'an Jiaotong University, Xi'an, Shaanxi 710068, P.R. China
}

Received November 21, 2015; Accepted June 22, 2016

DOI: $10.3892 /$ ijmm.2016.2662

\begin{abstract}
DNA binding protein A (dbpA) belongs to the Y-box binding protein family and has been reported to play an important role in carcinogenesis. Our previous study demonstrated that the knockdown of $\mathrm{dbpA}$ in gastric cancer cells inhibited cell proliferation by modulating the cell cycle. However, the role of $\mathrm{dbpA}$ in human colorectal cancer (CRC) remains unclear. In this study, immunohistochemical (IHC) staining and clinicopathological parameter analysis were employed to detect dbpA expression in 44 paired CRC samples and 7 CRC cell lines. Lentivirus-mediated short hairpin RNA (shRNA) was used to silence $\operatorname{dbpA}$, and the effects of dbpA knockdown on cell proliferation were determined by MTT assay, colony formation assay and flow cytometry. Furthermore, a xenograft model was established to observe tumor growth in vivo. Functional analysis indicated that $\mathrm{dbpA}$ was overexpressed in the CRC tissues and cell lines, and a high dbpA expression was associated with the depth of invasion $(p<0.001)$, the degree of differentiation $(p<0.001)$, lymphatic metastasis $(p<0.001)$ and vessel invasion $(\mathrm{p}<0.001)$. The suppression of $\mathrm{dbpA}$ expression resulted in decreased cell proliferation in vitro and tumor growth in vivo, and it induced cell cycle arrest and promoted the apoptosis of the CRC cells. As a whole, our findings illustrate the crucial role of $\mathrm{dbpA}$ in colorectal tumorigenesis. Thus, $\mathrm{dbpA}$ may be used as a novel and potent therapeutic target in CRC.
\end{abstract}

\section{Introduction}

Colorectal cancer (CRC) is among the most common malignancies and is becoming a leading cause of cancer-related mortality worldwide (1). Although the efficacy of multiple

Correspondence to: Professor Chang Liu, Department of Hepatobiliary Surgery, The First Affiliated Hospital, College of Medicine, Xi'an Jiaotong University, 277 West Yanta Road, Xi'an, Shaanxi 710061, P.R. China

E-mail: eyrechang@126.com

Key words: DNA binding protein A, lentivirus-mediated shRNA, colorectal cancer, proliferation, apoptosis drug treatments, surgical treatments and chemotherapy have extensively improved (2), CRC is still considered as a complex and difficult disease to deal with (3). In recent years, studies have focused on specific molecular targets, such as epidermal growth factor receptor (EGFR) and vascular endothelial growth factor (VEGF) to predict the progression of CRC $(4,5)$. However, due to the heterogeneous characteristics of CRC, there is still controversy regarding the optimal treatment strategy for CRC. Thus, in order to develop more effective targeting agents against CRC, the identification of novel molecules is urgently required (6).

Human DNA binding protein A (dbpA), a member of the Y-box binding protein family, contains a highly conserved DNA binding domain, named the cold shock domain (CSD) $(7,8)$. This family of proteins appears to play a critical role in cell proliferation and growth, transcriptional and translational regulation, DNA replication, drug resistance, the cell cycle and malignancy $(9,10)$. dbpA can bind to EGFR, proliferating cell nuclear antigen, thymidine kinase and DNA polymerase $(11,12)$ to participate in cellular activities. Previously, Tobita et al suggested that $\mathrm{dbpA}$ induced carcinogenesis by regulating the expression of cellular genes, such as insulin-like growth factor binding protein-1 (IGFBP-1) and carbonic anhydrase 3 (Car3) in dbpA-transgenic mice (13). Furthermore, dbpA has been reported as a prognostic marker for the advanced stages of and for the poor prognosis of hepatocellular carcinoma by enhancing cell proliferation and transformation (14,15). Our previous study demonstrated that dbpA played a crucial role in the development of gastric cancer by regulating the expression of E-cadherin, $\beta$-catinen, adenomatous polyposis coli (APC) and cyclin D1 (16). These findings indicate the significance of $\mathrm{dbpA}$ in the development of malignant diseases.

In the present study, to illustrate the role of $\mathrm{dbpA}$ in $\mathrm{CRC}$, the expression of $\mathrm{dbpA}$ in $\mathrm{CRC}$ tissues and cell lines was examined. The effects of dbpA on CRC cells were investigated by lentivirus-mediated short haripin RNA (shRNA) interference both in vitro and in vivo. Our findings indicate that $\mathrm{dbpA}$ is a vital driver of human $\mathrm{CRC}$ and that the knockdown of dbpA markedly reduces cell proliferation in vitro and decreases tumorigenesis in vivo. Our study may provide scientific evidence for the further development of reliable molecular biomarkers for CRC. 


\section{Materials and methods}

Clinical specimen collection. Fresh colorectal tumor and adjacent normal tissues were obtained from 44 patients who received surgery from May 2012 to July 2014 at the Department of General Surgery, the Third Affiliated Hospital of Xi'an Jiaotong University, Xi'an, China. We only collected the clinical data of patients that were complete and from patients who had not received any radiotherapy or chemotherapy prior to surgery. All specimens were classified according to the TNM staging system enacted by International Union Against Cancer (UICC) and American Joint Committee on Cancer (AJCC). The correlations between dbpA expression and clinicopathological parameters in CRC were analyzed by Pearson's Chi-square test. Written informed consent was obtained from all the patients, and this study was approved by the Human Ethics Committee of the Third Affiliated Hospital of Xi'an Jiaotong University and all experiments were performed in accordance with the 1964 Helsinki declaration and its later amendments.

Immunohistochemical (IHC) staining. The colorectal tissues obtained from patients were fixed immediately with $4 \%$ paraformaldehyde overnight at $4^{\circ} \mathrm{C}$, and were then embedded in paraffin and sectioned (4- $\mu \mathrm{m}$-thick) onto slides. The sections were baked at $60^{\circ} \mathrm{C}$ for $2 \mathrm{~h}$, deparaffinized by two changes of xylene, and rehydrated in graded alcohol solutions. For antigen retrieval, the sections were heated in $20 \mathrm{mmol} / 1$ sodium citrate $(\mathrm{pH} 6.0)$ at $95^{\circ} \mathrm{C}$ for $15 \mathrm{~min}$. The slides were treated then with $3 \% \mathrm{H}_{2} \mathrm{O}_{2}$ to block endogenous peroxidase activity. Subsequently, the slides were incubated overnight at $4^{\circ} \mathrm{C}$ with the rabbit polyclonal anti-dbpA antibody (ab48952; Boster Biological Technology, Ltd., Wuhan, China) at a 1:500 dilutions. Subsequently, the sections were incubated with HRP polymer (1:500; ab6721; Boster Biological Technology, Ltd.) for $30 \mathrm{~min}$ at room temperature and DAB mix (Tiangen Biotechnology, Beijing, China) was applied for staining. Two independent pathologists blinded to the patient data evaluated the scores of the IHC results. The scoring criteria was based on the percentage of dpbA-positive cells in the tumor tissue as follows: - $(<10 \%)$ was considered as no staining; $+(11-40 \%)$ was considered weak staining; $++(41-70 \%)$ was considered moderate staining; and +++ (71-100\%) was considered strong staining. If a discrepancy was exited between the scores, the specimens would be re-examined by both researchers together.

Cell lines and cell culture. The human CRC cell lines, LoVo, SW480, RKO, HT-29, DLD-1, SW1463 and SW620, were obtained from the First Affiliated Hospital of the Medical College of Xi'an Jiaotong University. The normal colorectal mucosa cell line, FHC, and the 293T cell line were purchased from the Animal Center of the Fourth Military Medical University, Xi'an, China. All the cells were cultured in Dulbecco's modified Eagle's medium (DMEM) supplemented with $10 \%$ fetal bovine serum (FBS) at $37^{\circ} \mathrm{C}$ with $5 \% \mathrm{CO}_{2}$.

dbpA RNAi lentivirus design and packaging. The shRNA sequences were designed for $\mathrm{dbpA}$ as follows: sense, 5'-AGA CGUGGCUACUAUGGAATT-3' and antisense, 5'-UUCCAU AGUAGCCACGUCUGT-3' in accordance with our previous study (16). The negative control shRNA were randomly sequenced and homology with the dbpA sequence was avoided using the Blast website (https://blast.ncbi.nlm.nih.gov/Blast. cgi). The shRNAs were then cloned into the pGV115 entry vector (Invitrogen Life Technologies, Carlsbad, CA, USA) and identified by PCR and DNA sequencing. Lentiviruses were produced and packaged using the 293T cells following co-transfection with pGV115 entry vector carrying dbpAshRNA (shRNA-dbpA-Lv) or scrambled shRNA (shNCdbpA-Lv) and pHelper plasmids, according to the lentivirus packaging protocol (Genechem Co., Ltd., Shanghai, China). The lentivirus contained the green fluorescent protein (GFP) and viral titers were then measured by GFP-positive cell counts under the observation of a fluorescence microscope (Olympus, Tokyo, Japan).

Infection of SW620 cells with shRNA-dbpA-Lv. The SW620 cells were seeded at $5 \times 10^{5}$ cells/well in 6-well plates and incubated for $24 \mathrm{~h}$ at $37^{\circ} \mathrm{C}$ with $5 \% \mathrm{CO}_{2}$. The cells were infected with shRNA-dbpA-Lv (GenePharma, Shanghai, China) at a multiplicity of infection (MOI) of 10. The knockdown efficiency of dbpA was evaluated at $72 \mathrm{~h}$ by the percentage of GFP-positive cells $(>50 \%)$. Cells were also infected with the shNC-dbpA-Lv plasmid as negative controls (NC). Cells transfected with empty vector were used as controls (CON). After infection at $120 \mathrm{~h}$, the cells were harvested and prepared for reverse transcriptionquantitative PCR (RT-qPCR) and western blot analysis.

RNA extraction and RT-qPCR. RNA from the cell cultures was extracted using TRizol reagent (Gibco Life Technologies, Beijing, China) according to the manufacturer's instructions. First strand cDNA was synthesized from $5 \mu \mathrm{g}$ of total RNA using SuperScript II RT $200 \mathrm{U} / \mu \mathrm{l}$ (Invitrogen). dbpA mRNA expression was evaluated by qPCR on an ABI 7500 Real-Time PCR System (Applied Biosystems Life Technologies, Beijing, China) with SYBR-Green PCR core reagents. GAPDH was used as the input reference. The sequences of the primers used wereasfollows: dbpAsense, 5'-CGTCGCTCACGGGTCTTA-3' and antisense, 5'-CCTGA AGTTGTGCTCCCTCT-3'; GAPDH sense, 5'-TGACTTCAACAGCGACACCCA-3' and antisense, 5'-CACCCTGTTGCTGTAGCCAAA-3'. RT-qPCR was performed in triplicate and the results are presented as the Ct values, defined as the threshold PCR cycle number at which an amplified product is first detected. The mean $\mathrm{Ct}$ value was calculated, and the $\Delta \mathrm{Ct}$ value was determined as the mean $\mathrm{Ct}$ value for the target gene minus the mean $\mathrm{Ct}$ value for GAPDH.

Western blot analysis. The cells were collected, washed with PBS and then lysed in lysis buffer containing $100 \mathrm{mM}$ Tris- $\mathrm{HCl}, \mathrm{pH} 7.5,0.5 \% \mathrm{NP}-40$ and protease inhibitor cocktail. The supernatant was collected after centrifugation (at 20,000 x g, for $15 \mathrm{~min}$, at $4^{\circ} \mathrm{C}$ ) and the protein concentrations were determined using a BCA protein assay kit (Pierce Biotechnology, Inc., Rockford, IL, USA). The cell lysate samples $(40 \mu \mathrm{g})$ were separated by $15 \%$ SDS-PAGE and transferred onto a polyvinylidene difluoride filter (Immobilon; Millipore, Bedford, MA, USA). After blocking with 5\% milk, the filter was incubated overnight with a primary rabbit polyclonal anti-dbpA antibody (ab48952; Boster Biological Technology, Ltd.) at 1:500 dilutions for $1 \mathrm{~h}$. The samples were then incubated with the HRP-conjugated secondary antibodies (ab6721) at 1:1,000, and the bands were detected by enhanced 
chemiluminescence (both from Amersham Biosciences, Piscataway, NJ, USA), and then analyzed using Quantity One software (Bio-Rad Laboratories, Inc., Hercules, CA, USA).

3-(4,5-Dimethylthiazol-2-yl)-2,5-diphenyltetrazolium bromide (MTT) assay. The SW620 cells transfected with shRNAdbpA-Lv or shNC-dbpA-Lv for 1 to 5 days, respectively, followed by diluting and seeding at a density of $2 \times 10^{3}$ cells/ $\mathrm{ml}$ into a 96 -well plate for $24 \mathrm{~h}$. MTT solution was added to each well to a final concentration of $5 \mathrm{mg} / \mathrm{ml}$ and culture was continued for $4 \mathrm{~h}$ at $37^{\circ} \mathrm{C}$. The supernatant mixed with MTT was removed and DMSO was added into each well. The OD data were analyzed once daily for 5 days using an ELISA reader (Bio-Rad Laboratories) at a wavelength of $490 \mathrm{~nm}$.

Colony formation assay. The SW620 cells transfected with shRNA-dbpA-Lv or shNC-dbpA-Lv were plated into 6-well plates $\left(2,000\right.$ cells/well) and incubated for 14 days at $37^{\circ} \mathrm{C}$, with the medium replaced every 3 days. After cultivating for 14 days, the cells were washed with PBS, fixed with $4 \%$ paraformaldehyde for $30 \mathrm{~min}$ at room temperature and stained with Giemsa (Tiangen Biotechnology) for $15 \mathrm{~min}$. The number of colonies containing $>50$ cells was counted under a microscope (CKX53; Olympus).

Cell cycle analysis. The effect of shRNA-dbpA-Lv on the cell cycle distribution was determined by flow cytometry. The cells were suspended at the concentration of $1 \times 10^{6}$ and centrifuged at 1,500 rmp for $5 \mathrm{~min}$ twice, then resuspended with $100 \mu 1 \mathrm{PBS}$ and fixed with $70 \%$ ice-cold ethanol at $4^{\circ} \mathrm{C}$ overnight. The cells were washed with PBS and resuspended in $1 \mathrm{ml}$ PBS containing $50 \mu \mathrm{g} / \mathrm{ml}$ PI and $100 \mu \mathrm{g} / \mathrm{ml}$ RNase A for $1 \mathrm{~h}$ in the dark at $4^{\circ} \mathrm{C}$. The cell cycle was analyzed using a FACSCalibur flow cytometer (Becton-Dickinson, San Jose, CA, USA) at $72 \mathrm{~h}$ after transduction. The proportions of cells in the G2/M, S, and G0/G1 phases were analyzed using special software FlowJo software (Tree Star, Inc., Ashland, OR, USA).

Apoptosis analysis. SW620 cells transfected shRNA-dbpA-Lv or shNC-dbpA-Lv were collected and washed with PBS twice. Following centrifugation (at $100 \mathrm{x}$ g, for $5 \mathrm{~min}$, at $4^{\circ} \mathrm{C}$ ), the cells were resuspended with $1 \mathrm{X}$ staining buffer at the concentration of $1 \times 10^{6}$ cells $/ \mathrm{ml}$, and the cells were then dyed with $5 \mu \mathrm{l}$ Annexin V-APC in the dark at room temperature for $15 \mathrm{~min}$. Flow cytometry was performed using a FACSCalibur flow cytometer (Becton-Dickinson) and analysis was performed with FlowJo software (Tree Star, Inc.).

Tumorigenicity assay. BALB/C nude mice (4 weeks old) were purchased from the Animal Center of the Fourth Military Medical University. Living SW620 cells were detected and harvested after mixing with $4 \%$ trypan blue, then washed and resuspended in PBS at $4^{\circ} \mathrm{C}$. The animals were randomly divided into 3 groups $(n=10)$ as follows: the mice subcutaneously injected with dbpA-shRNA (KD), those injected with negativeshRNA (NC) and those injected with normal cells (empty vector-transfected cells, CON). Each mouse was subcutaneously injected with $1 \times 10^{6}$ cells on the right side of axilla. The standard of tumorigenesis was based on a tumor diameter of $\geq 3 \mathrm{~mm}$. Tumor sizes [volume $\left(\mathrm{mm}^{3}\right)=$ width $\left(\mathrm{mm}^{2}\right) / 2 \times$ length $(\mathrm{mm})$ ]
Table I. Expression of dbpA in colorectal tumor and adjacent normal tissue samples.

\begin{tabular}{lcrcr}
\hline & Total & $\operatorname{DbpA}(-)$ & $\operatorname{DbpA}(+)$ & P-value \\
\hline Tumors & 44 & $9(20.5 \%)$ & $35(79.5 \%)$ & $0.001^{\mathrm{a}}$ \\
Normal tissues & 44 & $40(90.9 \%)$ & $4(9.1 \%)$ & \\
\hline
\end{tabular}

${ }^{\mathrm{a}}$ Indicates statistical significance. dbpA, DNA binding protein A.

were measured using calipers every 7 days. Furthermore, the physical conditions of the mice were monitored as follows: the thickness of subcutaneous fat of the mice was measured by calipers after pinching with fingers; the body weight of the mice was recorded using an electronic scale (Dongyi Biotechnology, Beijing, China). All mice were euthanized by cervical dislocation at 35 days post-inoculation, and the separated and complete tumors were collected and disposed for further analysis. The animal experiment was reviewed and approved by the Animal Care and Use Committee of Xi'an Jiaotong University.

Statistical analysis. Statistical analyses were performed using SPSS 12.0 software. Each experiment was repeated 3 times, unless otherwise indicated. All data are presented as the means \pm standard deviation (SD). Comparisons between groups were carried out using the unpaired Student's t-test. A p-value of $<0.05$ was considered to indicate a statistically significant difference for all analyses.

\section{Results}

Expression of dbpA is increased in CRC tumor samples and varies in CRC cell lines. To explore the role of $\mathrm{dbpA}$ in human colorectal tumors, we began by analyzing the expression of $\mathrm{dbpA}$ in human CRC tissues. Paired colorectal tumor samples were obtained from 44 patients and dbpA expression was examined by IHC staining. Approximately 79.5\% (35/44; $\mathrm{p}<0.001)$ of the CRC samples exhibited a positive dbpA expression, while $20.5 \%(9 / 44)$ of the CRC samples were negative for dbpA expression; only 9.1\% (4/44) of the adjacent normal tissue samples exhibited a positive dbpA expression (Table I). We also found that $\mathrm{dbpA}$ was predominantly expressed in the cytoplasm of the CRC cells rather than in the nucleus or the cytomembrane region (Fig. 1A, top panels). Furthermore, IHC analysis revealed that the level of $\mathrm{dbpA}$ expression correlated with the depth of invasion in CRC. With the progression of the depth of invasion of the CRC tumor tissues (T1 to T4), the level of dbpA expression gradually increased (Fig. 1B, bottom panels). Collectively, the dbpA expression levels were significantly higher in the CRC tissues than in the non-tumor tissues, which implies that dbpA may facilitate tumorigenesis in the colon.

In addition, the expression levels of $\mathrm{dbpA}$ were assessed in different CRC cell lines, namely in the RKO, SW480, LoVo, DLD-1, SW1463, HT-29, SW620 cells, and in the normal colorectal mucosa cell line, FHC. The results of RT-qPCR revealed that the mRNA expression levels of $\mathrm{dbpA}$ varied among the cell lines. In the CRC cell lines, the expression levels from lowest to highest were: LoVo, DLD-1, RKO, HT-29, 
SW1463, SW480 and SW620 cells (Fig. 1B); however, no dbpA expression was detected in the normal colorectal mucosa cell line, FHC, which was consistent with the results of western blot analysis (Fig. 1C). The SW620 cells were selected for use in futher experiments as they exhibited the highest expression of dbpA. Our findings indicated that dbpA is dominantly expressed in CRC tissues or cell lines rather than in normal colon tissues or cells, and the expression profile differs amongst CRC cell lines.

dbpA expression correlates with different clinicopathological parameters. Considering that the level of dbpA in CRC tissues was significantly higher than that in non-tumor tissues, the correlations between dbpA expression and clinicopathological parameters in CRC were analyzed by Pearson's Chi-square test. We subdivided the CRC-positive cases into 3 groups according to the criteria described in the Materials and methods. The percentage of dbpA weak expression (+) was $28.6 \%(10 / 35)$, moderate expression $(++)$ was $31.4 \%(11 / 35)$, and strong expression was $40.0 \%$ (14/35). Correlation analysis demonstrated that the strong expression of dbpA was significantly associated with the degree of differentiation $(p<0.001)$, the depth of invasion $(\mathrm{p}<0.001)$, lymphatic metastasis $(\mathrm{p}<0.001)$, vessel invasion $(\mathrm{p}<0.001)$ and the TNM stage $(\mathrm{p}<0.05)$, but not with age, gender, tumor size, stage, type, distant metastasis, or surgical method (Table II). Therefore, we hypothesized that dbpA may be considered as a potential unfavorable prognostic biomarker for patients with CRC.

Effect of shRNA on dbpA expression in SW620 cells. Lentivirus-mediated shRNA inferference was applied to suppress the expression of dbpA in the SW620 cells. The efficiency of shRNA-dbpA-Lv or shNC-dbpA-Lv was detected by fluorescent microscopy in the infected cells. The results revealed that a great proportion of cells was infected with the shRNA (Fig. 2A); the infected percentage was $>90 \%$ (data not shown).

RT-qPCR and western blot analysis were performed to further examine the silencing efficiency of the shRNA against dbpA. At $72 \mathrm{~h}$ post-transfection, the mRNA level of dbpA in the shRNA-dbpA-Lv group (KD) was significantly decreased when compared to the shNC-dbpA-Lv (NC) or control (CON) group ( $<<0.01$; Fig. 2B). Western blot analysis also verified that the protein expression of dbpA was markedly decreased in the $\mathrm{KD}$ group in comparison to the $\mathrm{CON}$ or $\mathrm{NC}$ groups (Fig. 2C); no significant difference was observed between the $\mathrm{NC}$ and CON group. Therefore, our results indicated that lentivirusmediated dpbA RNAi successfully and efficiently suppressed dbpA expression in the SW620 cells.

Silencing of dbpA suppresses the proliferation of SW620 cells. MTT assay was employed to assess the effects of the silencing of dbpA expression on the proliferation of the SW620 cells in vitro. Cell proliferation was analyzed by MTT assay once daily for 5 days. We found that the cells transfected with shRNA-dbpA-Lv proliferated more slowly than the cells in the $\mathrm{NC}$ and CON groups from 3 days onwards. In addition, on the 4 th and 5th day, the proliferation rates in the KD group were significantly decreased $(p<0.05$ and $p<0.01$, respectively) when compared with the NC group (Fig. 3A).
Table II. Association between dbpA expression and clinicopathologic factors in patient with CRC.

The level dbpA expression

\begin{tabular}{lllll}
\cline { 2 - 3 } Parameters & + & ++ & +++ & P-value \\
Age (years) & 8 & 6 & 9 & 0.431 \\
$>60$ & 2 & 5 & 5 & \\
$\quad 60$ & & & & \\
Gender & 7 & 7 & 8 & 0.708 \\
$\quad$ Male & 3 & 4 & 6 & \\
$\quad$ Female & & & & \\
Tumor size $(\mathrm{cm})$ & 6 & 5 & 4 & 0.528 \\
$>4$ & 4 & 6 & 10 & \\
$\leq 4$ & & &
\end{tabular}

Degree of

differentiation

Well

Moderate

Poor

Invasion depth

T1

$\mathrm{T} 2$

$\mathrm{T} 3$

$\mathrm{T} 4$

Tumor site

Colon

Rectum

TNM stage

I+II

III+IV

Lymphatic metastasis

NO

N1

N2

$\begin{array}{llll}7 & 2 & 0 & <0.001^{\mathrm{b}} \\ 3 & 9 & 5 & \\ 0 & 0 & 9 & \end{array}$

$<0.001^{\text {b }}$

$\begin{array}{lll}0 & 0 & 0 \\ 1 & 0 & 0\end{array}$

$\begin{array}{lll}5 & 5 & 2\end{array}$

$4 \quad 6 \quad 12$

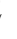

12

0.900

$\begin{array}{lll}5 & 2 & 6 \\ 5 & 9 & 8\end{array}$

8

$<0.05^{\mathrm{a}}$

$\begin{array}{lll}5 & 4 & 0\end{array}$

14

Distant metastasis

M0

M1

Tumor type

Infiltrate type

Ulcerative type

Protrude type

5

7

14

$\begin{array}{lll}7 & 5 & 1\end{array}$

1

$<0.001^{\text {b }}$

$\begin{array}{lll}3 & 4 & 10\end{array}$

$\begin{array}{lll}0 & 2 & 3\end{array}$

Vessel invasion

0

1

$\begin{array}{rrr}9 & 11 & 11 \\ 1 & 0 & 3\end{array}$

0.485

3

0.788

$\begin{array}{rrr}1 & 0 & 2 \\ 9 & 9 & 12\end{array}$

020

2

0

$10 \quad 10 \quad 4$

4

10

Surgical method

$\begin{array}{lllll}\text { Dixon } & 5 & 7 & 7 & 0.670 \\ \text { Mile's } & 0 & 2 & 7 & \\ \text { Hemicolectomy } & 5 & 2 & 0 & \end{array}$

a,b Indicates statistical significance and high statistical significance, respectively. CRC, colorectal cancer; dbpA, DNA binding protein A. 
A

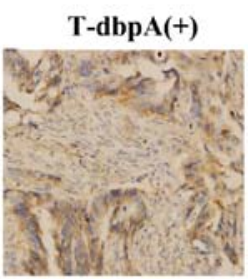

T1

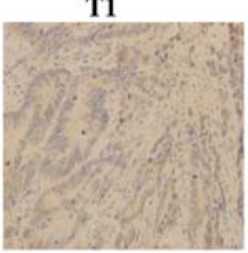

B

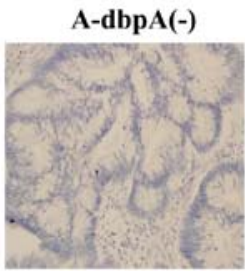

T2
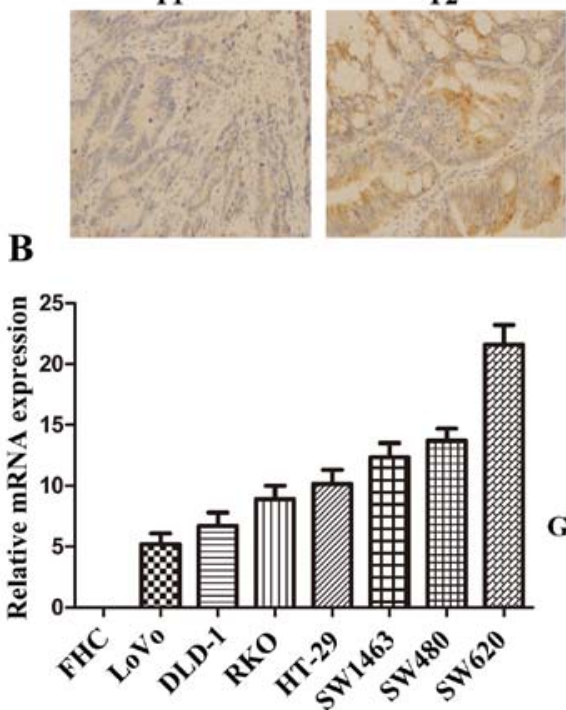

$\operatorname{A-dbpA}(+)$

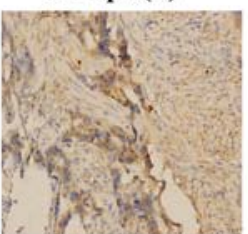

T3

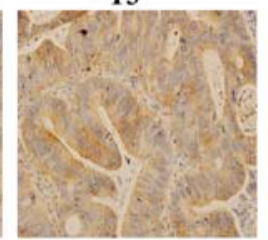

C-dbpA(+)

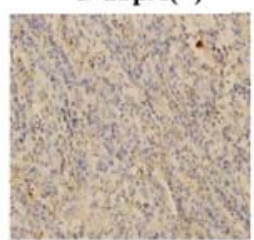

T4

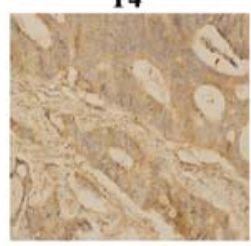

C

$\operatorname{dbpA}$

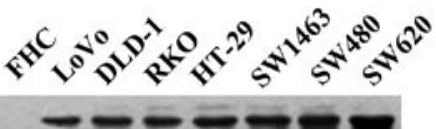

GAPDH

Figure 1. Expression of DNA binding protein A (dbpA) in tissues obtained from patients with colorectal cancer (CRC) and various CRC cell lines using immunohistochemistry (IHC). T, tumor tissues; A, adjacent normal tissues; C, cytoplasm; and T1-T4, invasion depth. (A) dbpA expression in tumors and adjacent normal tissues (x100 magnification). (B) The levels of dbpA expression in different invasion depths (x100 magnification). RT-qPCR analysis of the mRNA expression of the dbpA in various human CRC cell lines. (C) Western blot analysis of dbpA protein levels. GAPDH was used as an internal control. Data are shown as the means \pm standard deviation $(\mathrm{SD})$.

A
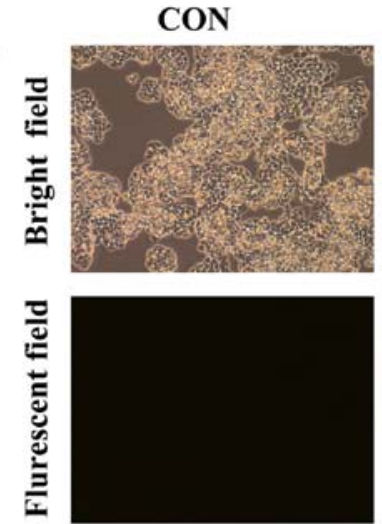

B

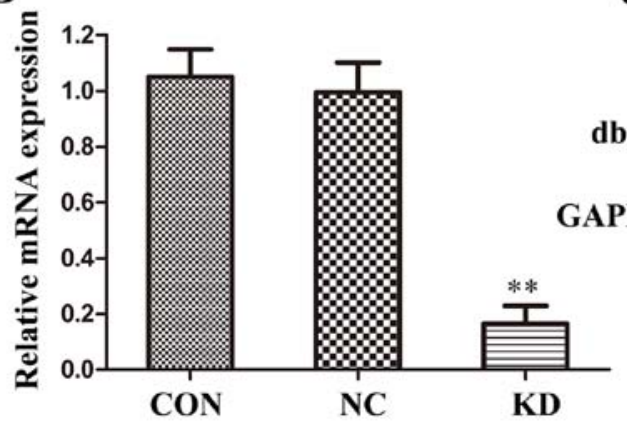

NC
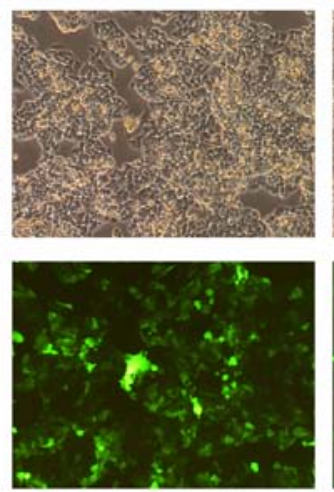

C
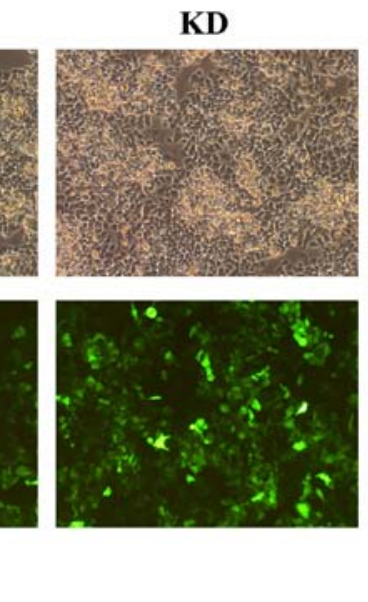

$\operatorname{dbpA}$

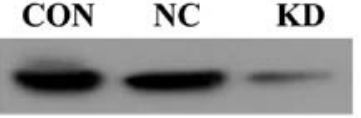

APDH

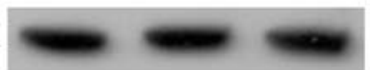

Figure 2. DNA binding protein A (dbpA) knockdown by lentiviral-mediated RNAi. (A) Representative images of SW620 cells transfected with shRNA-dbpA-Lv, shNC-dbpA-Lv, or untransfected cells (CON) for $72 \mathrm{~h}$ (x100 magnification). GFP expression indicated that the cells had been successfully infected with the shRNA. (B) RT-qPCR analysis of dbpA mRNA levels in cells treated as in (A). (C) Western blot analysis showing dbpA protein levels in cells treated as in (A). GAPDH was used as an internal control. Data are shown as the means \pm standard deviation (SD); * $p<0.01$ vs. NC. 

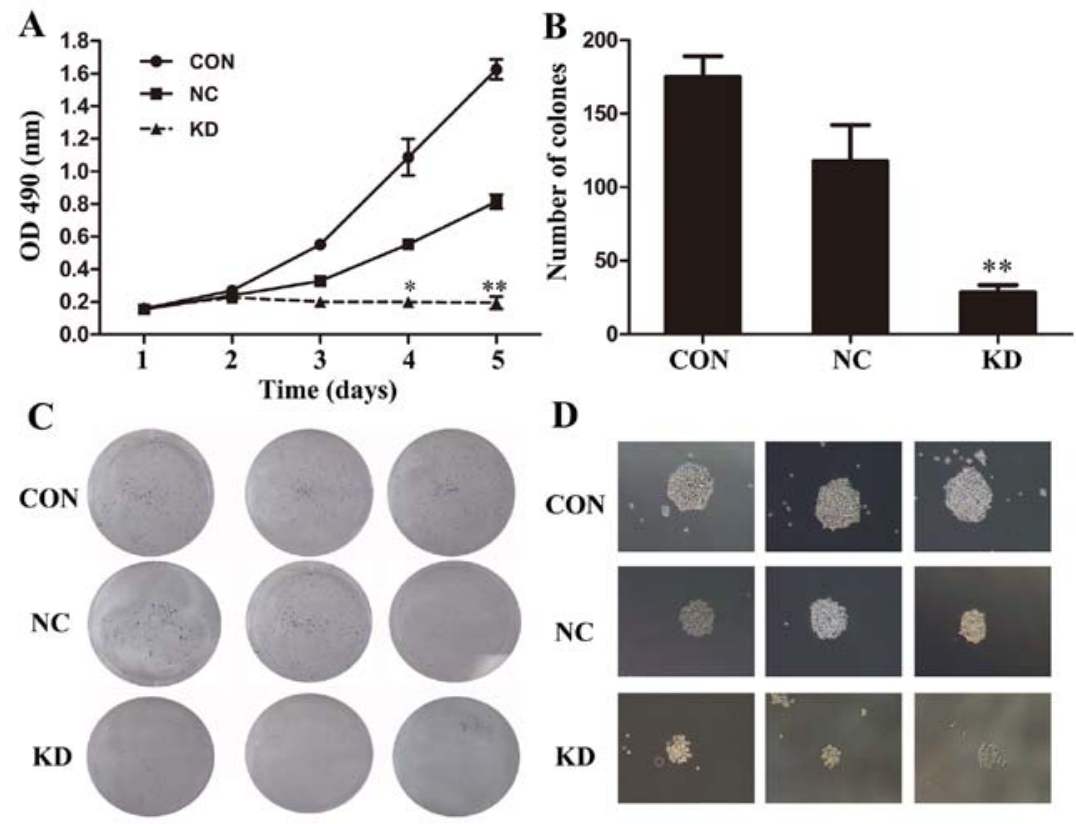

Figure 3. Effect of DNA binding protein A (dbpA) silencing on SW620 cell proliferation. (A) MTT assay measuring cell proliferation of lentivirus-transfected SW620 cells. (B) Quantification of clone number in SW620 cells transfected with shRNA-dbpA-Lv, shNC-dbpA-Lv, or empty vector-transfected cells (CON). (C) Representative images of colony formation assay for SW620 cells in a 6-well plate. (D) Representative images per colony for SW620 cells under a bright microscope. Data are shown as the means \pm standard deviation $(\mathrm{SD}) ;{ }^{*} \mathrm{p}<0.05,{ }^{* *} \mathrm{p}<0.01 \mathrm{vs}$. NC.

Downregulation of dbpA inhibits the colony-forming ability of SW620 cells. Furthermore, when the SW620 cells were transfected with dbpA-shRNA, negative-shRNA, or the empty vyector-transfected cells were incubated for 14 days, the colony-forming capacity of the SW620 cells was determined. The number of colonies was counted following Giemsa staining. Our results revealed that the number cell colonies in the KD group declined significantly $(\mathrm{p}<0.01)$ in comparison with the control group (Fig. 3B and C). In addition, the size of the colonies was markedly reduced in the KD group compared with the control group (Fig. 3D). On the whole, these findings indicated that the silencing of $\mathrm{dbpA}$ by lentivirus-mediated RNAi efficiently suppressed SW620 cell proliferation in vitro.

Silencing of dbpA expression induces SW620 cell cycle arrest. Flow cytometry was adopted to detect cell cycle progression in the dbpA-shRNA-transfected cells. The results revealed that compared with the $\mathrm{NC}$ group, the proportion of dbpA-shRNA-transfected cells in the $\mathrm{S}$ phase was significantly decreased from 43.92 to 25.19 ( $\mathrm{p}<0.01)$, while the ratio of cells in the G0/G1 phase was significantly increased from 35.24 to $54.45(\mathrm{p}<0.01)$, indicating that dbpA-shRNA interfered with the distribution of the cell cycle, leading to cell cycle arrest at the G1 phase in the KD group (Fig. 4). However, no significant difference in the number of cells in the $S$ phase or the G0/G1 phase was observed between the cells in the CON group and NC group. Thus, knocking down dbpA expression suppressed SW620 cell proliferation by leading to cell cycle arrest at the G0/G1 phase.

Knockdown of dbpA expression promotes the apoptosis of SW620 cells. The balance between the cell cycle and cell apoptosis is the key premise to maintain tumorigenesis in patients (17). Hence, we considered it necessary to examine the effects of dbpA silencing on SW620 cell apoptosis in this study. Apoptosis was determined by FITC-labeled Annexin V/ PI double staining and flow cytometric analysis. The results revealed that the silencing of the expression of dbpA in the KD group significantly increased the apoptotic rate when compared with the NC group ( $<<0.01$; Fig. $5 \mathrm{~A}$ and B). These results indicated that the silencing of dbpA expression promoted cell apoptosis which correlated with the inhibitory effects on cell proliferation.

Silencing dbpA decreases tumorigenesis in CRC in vivo. Since the knocking down of dbpA exerted an inhibitory effect on tumorigenesis in vitro, we considered it crucial to assess its effects in vivo. A xenograft tumor model was established by subcutaneously injecting normal cells (empty vector-transfected cells), negative control- or dbpA-shRNA-transfected SW620 cells into nude mice. After 14 days, the average tumor volume in the mice in the KD group was markedly smaller than that in the NC or CON groups (Fig. 6A). Furthermore, through monitoring the physical conditions of the injected mice, at 30 days post-treatment, the mice in the $\mathrm{NC}$ and $\mathrm{CON}$ groups reserved less subcutaneous fat and exhibited more weight loss than the mice in the KD group. At the 35th day, during the process of tumor isolation, we found that in the $\mathrm{NC}$ and $\mathrm{CON}$ groups, the surfaces of tumors appeared uneven with ulcerations and bleeding, while in the KD group, the tumors appeared more complete and had smooth outer members (Fig. 6C). The average weight of the tumors in the KD group was significantly smaller than that in the NC group, with the tumor inhibition rate at $93.97 \%(\mathrm{p}<0.05)$ (Fig. 6B and C). However, no difference was observed either in tumor volume or tumor weight between the $\mathrm{CON}$ group and $\mathrm{NC}$ group. In addition, western 

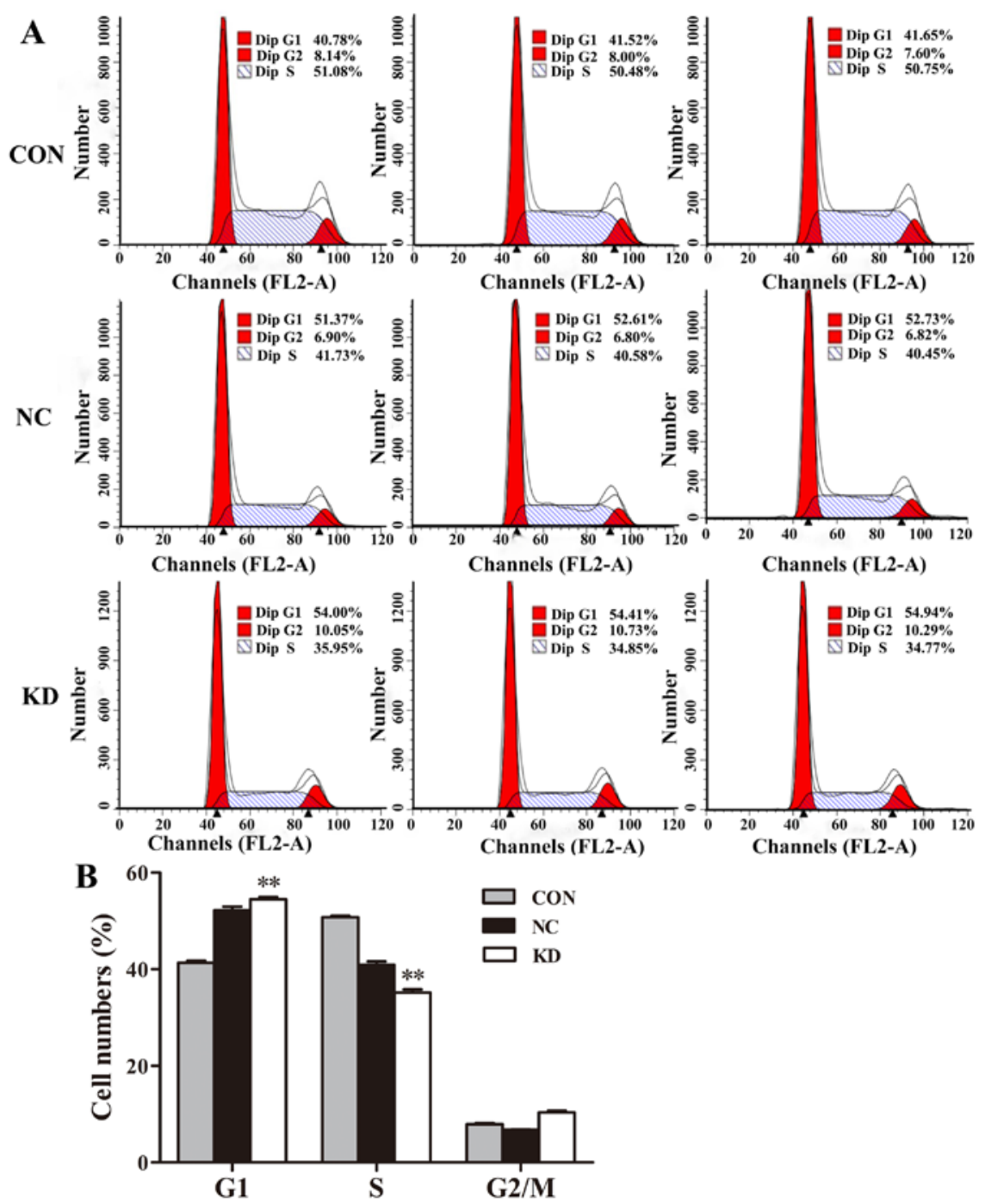

Figure 4. Effect of DNA binding protein A (dbpA) silencing on cell cycle progression in SW620 cells. (A) Representative images of cell cycle analysis at $72 \mathrm{~h}$ by flow cytometry. (B) Cell cycle distribution in SW620 cells transfected with shRNA-dbpA-Lv, shNC-dbpA-Lv, or empty vector. Data are shown as the means \pm standard deviation $(\mathrm{SD}) ;{ }^{* *} \mathrm{p}<0.01$ vs. NC.

blot analysis verified that the protein expression of $\mathrm{dbpA}$ was downregulated in the KD group in comparison to the $\mathrm{NC}$ or CON groups (Fig. 6D). Therefore, our results demonstrated that the suppression of the expression of dbpA in the SW620 cells significantly inhibited tumor growth in vivo.

\section{Discussion}

dbpA as a member of the Y-box protein family, has aroused great interest in recent years and has been reported to be involved in the development of malignant tumors, such as hepatocellular carcinoma (18) and gastric cancer (16). dbpA regulate the proliferation of epithelial cells, accelerates inflammation-induced hepatocarcinogenesis, and plays a vital role in the pathogenesis and development of gastric cancer (16). Although dbpA is considered as an oncogene in several tumors, its pathogenic mechanisms of action in CRC remain unknown. Hence, in this study, we examined dbpA expression in tumor samples from patients with CRC, and clarified that dbpA was overexpressed in CRC tumor tissues compared to paired adjacent normal tissues. To the best of our knowledge, we provided the first evidence to verify that dbpA plays an important role in $\mathrm{CRC}$ tumorigenesis.

In this study, 35 CRC cases with various TNM stages (I-IV) were selected to investigate the association between the clinical characteristics of CRC tumors and dbpA expression levels. Our findings illustrated that $\mathrm{dbpA}$ expression positively correlated with the degree of differentiation, the depth of invasion, vessel invasion and an advanced TNM stage, which are all the key features to accelerate cancer development (19). Furthermore, IHC staining revealed that $\mathrm{dbpA}$ was mainly expressed in the cytoplasm and was associated with the progression of CRC in patients. Yasen et al had emphasized that both the cytoplasmic expression and the nuclear localization of $\mathrm{dbpA}$, as a significant prognostic marker, was responsible for the advanced stages of hepatocellular carcinoma (14). However, during our study, no dbpA expression was found in the nucleus in our CRC cases. These exiting data indicate that the localization of $\mathrm{dbpA}$ may alternate, depending on advanced cancer stages or different cancer types. Further studies with larger sample sizes are warranted in order to deeply investigate the variation of $\mathrm{dbpA}$ localization in CRC progression. 

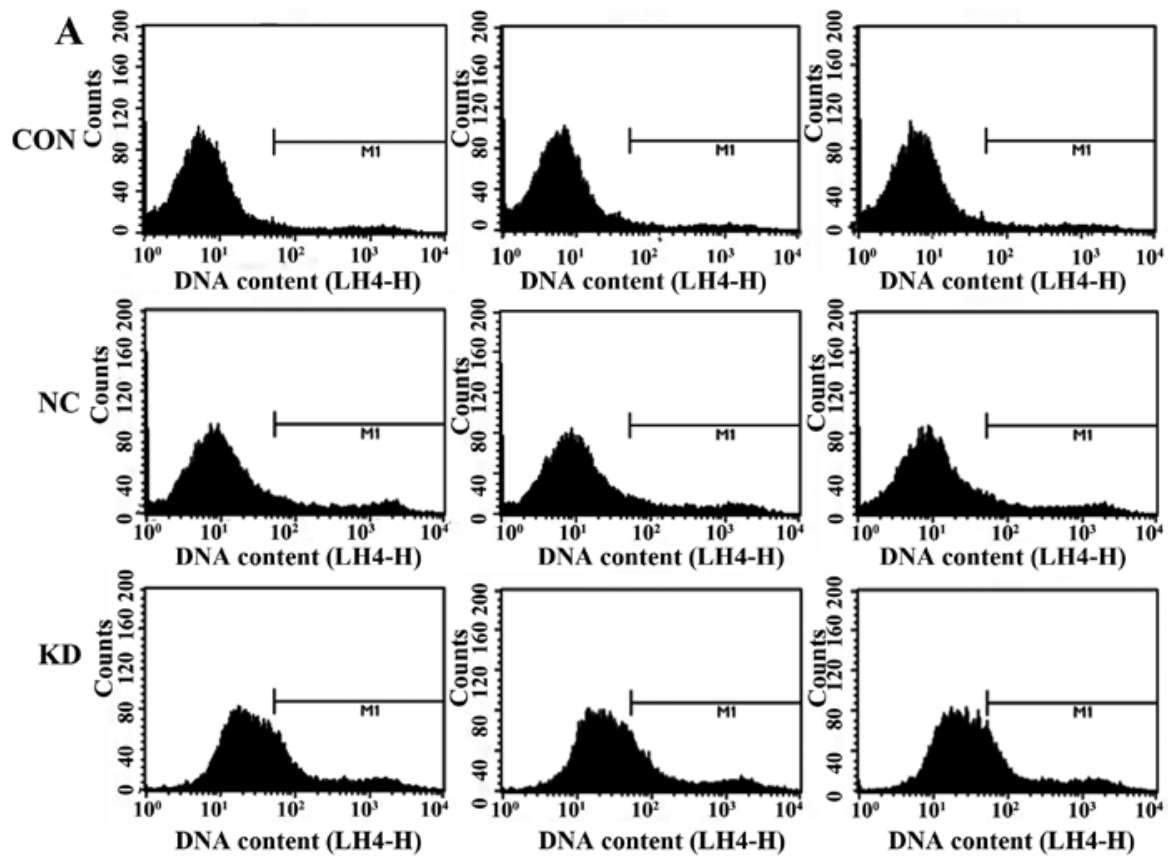

B

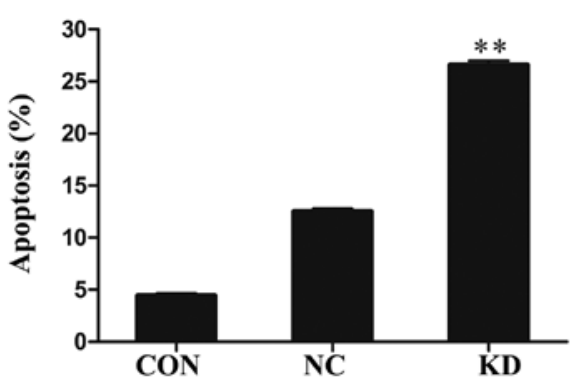

Figure 5. Apoptosis induction in SW620 cells transfected with short hairpin RNA (shRNA)-DNA binding protein A (dbpA)-Lv, shNC-dbpA-Lv, or empty vector. (A) Cell apoptosis was analyzed by flow cytometry. (B) Cell apoptosis in SW620 cells treated as in (A). Data are shown as the means \pm standard deviation (SD); ${ }^{* *} \mathrm{p}<0.01$ vs. NC.

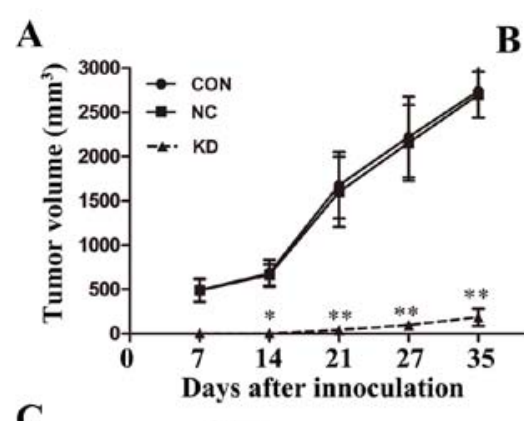

C

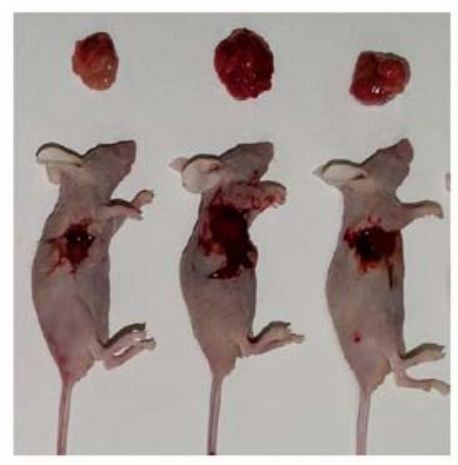

B

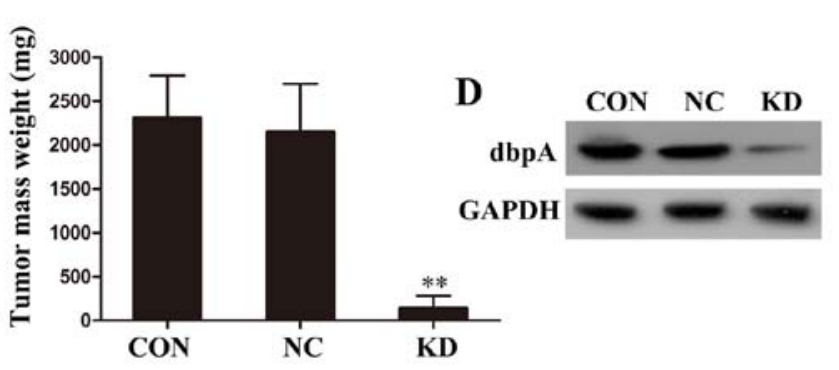

NC
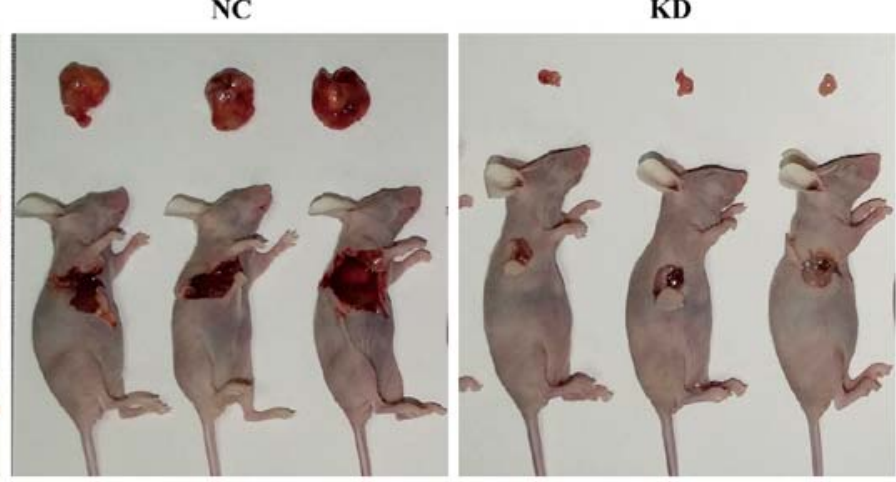

Figure 6. Effect of DNA binding protein A (dbpA) knockdown on tumorigenesis in nude mice. (A) Curve of tumor volume was assessed by caliper measurements. (B) Mass weight of tumor at the 35th day after inoculation. (C) Representative images of mice and tumors from each group. (D) dbpA expression was detected by western blot analysis from isolated tumors. GAPDH was used as an internal control. Data are shown as the means \pm standard deviation (SD); ${ }^{*}<<0.05$, ${ }^{* *} \mathrm{p}<0.01$ vs. NC. 
In the present study, we found that $\mathrm{dbpA}$ expression varied in different CRC cell lines. The SW620 cells derived from colorectal adenocarcinoma had the highest expression of $\mathrm{dbpA}$. Thus, for this reason, we selected these cells for use in our subsequent experiments. Small interference RNA and lentiviral vector-mediated RNAi have been extensively used as efficient tools to investigate the specific genes involved in abnormal cell proliferation and are regarded as promising therapeutic methods to deal with malignant tumors $(20,21)$. Lentivirus-mediated shRNA interference was conducted in this study to inhibit dbpA expression in the SW620 cells. As a result, the knockdown of dbpA suppressed SW620 cell proliferation by inducing cell cycle arrest in the G0/G1 phase in vitro. Furthermore, through establishing a xenograft model using nude mice, we found that $\mathrm{dbpA}$ silencing significantly inhibited tumor growth and tumorigenesis in vivo. Therefore, modulating $\mathrm{dbpA}$ expression resulted in changes in cell proliferation both in vitro and in vivo. Abnormal cell proliferation is considered as the key element in the progression of cancer (22). The occurrence of cell proliferation in physiological conditions is always regulated by specific molecular signaling pathways (23). For example, the duplicate progression of a cell is mediated by a group of proteins known as cyclins (24). Cyclins act in part with the cyclin-dependent kinases (CDKs) to phosphorylate key substrates that participate in each phase of the cell cycle (25). Researchers have reported that increased cyclin expression is frequently observed in human malignancies (26). For example, our previous study also proved that the silencing of $\mathrm{dbpA}$ suppressed the transcription of cyclin D1 and resulted in the inhibition of the proliferation of gastric cancer cells (16); this may also explain the similar results obtained in our present study.

Apoptosis is a natural way of removing aged cells from the living body (27), whereas under cancer conditions, the uncontrolled regulation of apoptotic signals assists cancer cells to escape from this programmed death and leads to abnormal proliferation (28). Various molecular signaling pathways have been found to be involved in this complex program, such as the B-cell lymphoma 2 (Bcl-2) signaling pathway (29), the heat shock protein signaling pathway (30) and the proteasome pathway (31). In this study, we confirmed that the silencing of dbpA significantly increased the apoptosis of SW620 cells, suggesting that $\mathrm{dbpA}$ may regulate apoptosis by activating or inactivating certain signaling pathways. The exact underlying molecular mechanisms responsible for the apoptosis observed by the silencing of $\mathrm{dbpA}$ in $\mathrm{CRC}$ requires further investigation.

In conclusion, our study confirmed that $\mathrm{dbpA}$ was overexpressed in CRC tissues and cell lines. The high expression level of dbpA closely correlated with certain clinicopathological parameters and tumor progression in CRC. Lentivirus-mediated RNAi of dbpA inhibited SW620 cell growth in vitro and tumorigenesis in vivo. Furthermore, the silencing of $\mathrm{dbpA}$ induced cell cycle arrest and promoted cell apoptosis. Hence, our findings illustrate the biological significance of $\mathrm{dbpA}$ in tumorigenesis in CRC and provide scientific evidence to develop a novel therapeutic target for the more effective treatment of patients with CRC. Moreover, further investigations are required in order to comprehensively reveal the intrinsic mechanisms of action of $\mathrm{dbpA}$ in CRC.

\section{Acknowledgements}

The present study was funded by grants from the National Natural Science Foundation of China (no. 81172363/H1617) and the Natural Science Foundation of Shaanxi Province (2014JM4089).

\section{Reference}

1. Siegel R, Desantis C and Jemal A: Colorectal cancer statistics, 2014. CA Cancer J Clin 64: 104-117, 2014.

2. Shi Q, Mandrekar SJ and Sargent DJ: Predictive biomarkers in colorectal cancer: usage, validation, and design in clinical trials. Scand J Gastroenterol 47: 356-362, 2012.

3. Compton CC: Colorectal carcinoma: diagnostic, prognostic, and molecular features. Mod Pathol 16: 376-388, 2003.

4. Custodio A and Feliu J: Prognostic and predictive biomarkers for epidermal growth factor receptor-targeted therapy in colorectal cancer: beyond KRAS mutations. Crit Rev Oncol Hematol 85: 45-81, 2013.

5. De Mattos-Arruda L, Dienstmann R and Tabernero J: Development of molecular biomarkers in individualized treatment of colorectal cancer. Clin Colorectal Cancer 10: 279-289, 2011.

6. Linnekamp JF, Wang X, Medema JP and Vermeulen L: Colorectal cancer heterogeneity and targeted therapy: a case for molecular disease subtypes. Cancer Res 75: 245-249, 2015.

7. Kudo S, Mattei MG and Fukuda M: Characterization of the gene for $\mathrm{dbpA}$, a family member of the nucleic-acid-binding proteins containing a cold-shock domain. Eur J Biochem 231: 72-82, 1995.

8. Petruzzelli R, Gaudino S, Amendola G, Sessa R, Puzone S, Di Concilio R, d'Urzo G, Amendolara M, Izzo P and Grosso M: Role of the cold shock domain protein $\mathrm{A}$ in the transcriptional regulation of HBG expression. Br J Haematol 150: 689-699, 2010.

9. Hasegawa SL, Doetsch PW, Hamilton KK, Martin AM, Okenquist SA, Lenz J and Boss JM: DNA binding properties of YB-1 and dbpA: binding to double-stranded, single-stranded, and abasic site containing DNAs. Nucleic Acids Res 19: 4915-4920, 1991.

10. Kohno K, Izumi H, Uchiumi T, Ashizuka M and Kuwano M: The pleiotropic functions of the Y-box-binding protein, YB-1. BioEssays 25: 691-698, 2003.

11. Wolffe AP: Structural and functional properties of the evolutionarily ancient Y-box family of nucleic acid binding proteins. BioEssays 16: 245-251, 1994.

12. Ladomery $\mathbf{M}$ and Sommerville J: A role for Y-box proteins in cell proliferation. BioEssays 17: 9-11, 1995.

13. Tobita H, Kajino K, Inami K, Kano S, Yasen M, Imamura O, Kinoshita $Y$ and Hino O: Gene expression profile of DNA binding protein A transgenic mice. Int J Oncol 29: 673-679, 2006.

14. Yasen M, Kajino K, Kano S, Tobita H, Yamamoto J, Uchiumi T, Kon S, Maeda M, Obulhasim G, Arii S, et al: The up-regulation of Y-box binding proteins (DNA binding protein A and Y-box binding protein-1) as prognostic markers of hepatocellular carcinoma. Clin Cancer Res 11: 7354-7361, 2005.

15. Arakawa Y, Kajino K, Kano S, Tobita H, Hayashi J, Yasen M, Moriyama M, Arakawa Y and Hino O: Transcription of dbpA, a $\mathrm{Y}$ box binding protein, is positively regulated by E2F1: implications in hepatocarcinogenesis. Biochem Biophys Res Commun 322: 297-302, 2004.

16. Wang GR, Zheng Y, Che XM, Wang XY, Zhao JH, Wu KJ, Zeng J, Pan CE and He DL: Upregulation of human DNA binding protein A (dbpA) in gastric cancer cells. Acta Pharmacol Sin 30: 1436-1442, 2009.

17. Evan GI and Vousden KH: Proliferation, cell cycle and apoptosis in cancer. Nature 411: 342-348, 2001.

18. Yasen M, Obulhasim G, Kajino K, Mogushi K, Mizushima H, Tanaka S, Tanaka H, Hino O and Arii S: DNA binding protein A expression and methylation status in hepatocellular carcinoma and the adjacent tissue. Int J Oncol 40: 789-797, 2012.

19. Fallowfield LJ and Fleissig A: The value of progression-free survival to patients with advanced-stage cancer. Nat Rev Clin Oncol 9: 41-47, 2011.

20. Guo W, Chen W, Yu W, Huang W and Deng W: Small interfering RNA-based molecular therapy of cancers. Chin J Cancer 32: 488-493, 2013. 
21. Sumimoto $\mathrm{H}$ and Kawakami Y: Lentiviral vector-mediated RNAi and its use for cancer research. Future Oncol 3: 655-664, 2007.

22. Von Wangenheim KH and Peterson HP: The role of cell differentiation in controlling cell multiplication and cancer. J Cancer Res Clin Oncol 134: 725-741, 2008.

23. Feitelson MA, Arzumanyan A, Kulathinal RJ, Blain SW, Holcombe RF, Mahajna J, Marino M, Martinez-Chantar ML, Nawroth R, Sanchez-Garcia I, et al: Sustained proliferation in cancer: mechanisms and novel therapeutic targets. Semin Cancer Biol 35 (Suppl): S25-S54, 2015.

24. Canavese M, Santo L and Raje N: Cyclin dependent kinases in cancer: potential for therapeutic intervention. Cancer Biol Ther 13: 451-457, 2012.

25. Węsierska-Gądek J and Maurer M: Promotion of apoptosis in cancer cells by selective purine-derived pharmacological CDK inhibitors: one outcome, many mechanisms. Curr Pharm Des 17: 256-271, 2011.

26. Casimiro MC, Velasco-Velázquez $\mathrm{M}$, Aguirre-Alvarado $\mathrm{C}$ and Pestell RG: Overview of cyclins D1 function in cancer and the CDK inhibitor landscape: past and present. Expert Opin Investig Drugs 23: 295-304, 2014.
27. Elmore S: Apoptosis: a review of programmed cell death. Toxicol Pathol 35: 495-516, 2007.

28. Ouyang L, Shi Z, Zhao S, Wang FT, Zhou TT, Liu B and Bao JK: Programmed cell death pathways in cancer: a review of apoptosis, autophagy and programmed necrosis. Cell Prolif 45: 487-498, 2012

29. Yating Q, Yuan Y, Wei Z, Qing G, Xingwei W, Qiu Q and Lili Y: Oxidized LDL induces apoptosis of human retinal pigment epithelium through activation of ERK-Bax/Bcl-2 signaling pathways. Curr Eye Res 40: 415-422, 2015.

30. Qi Z, Shen L, Zhou H, Jiang Y, Lan L, Luo L and Yin Z: Phosphorylation of heat shock protein 27 antagonizes TNF- $\alpha$ induced HeLa cell apoptosis via regulating TAK1 ubiquitination and activation of 38 and ERK signaling. Cell Signal 26: 1616-1625, 2014.

31. Mohammad RM, Muqbil I, Lowe L, Yedjou C, Hsu HY, Lin LT, Siegelin MD, Fimognari C, Kumar NB, Dou QP, et al: Broad targeting of resistance to apoptosis in cancer. Semin Cancer Biol 35 (Suppl): S78-S103, 2015. 